\title{
Recuperação da qualidade estrutural, pelo sistema plantio direto, de um Argissolo Vermelho
}

\author{
Soil structural quality restoration by no-tillage system of a Hapludalf \\ Carlos Alberto Flores ${ }^{\mathrm{I}}$ Dalvan José Reinert ${ }^{\mathrm{II}}$ José Miguel Reichert ${ }^{\mathrm{II}}$ \\ Jackson Adriano Albuquerque ${ }^{\mathrm{III}}$ Eloy Antonio Pauletto ${ }^{\mathrm{IV}}$
}

\section{RESUMO}

Solos com quantidades elevadas de areia no horizonte A são pouco resistentes aos processos de degradação, dependendo do sistema de cultivo utilizado. Este trabalho objetivou avaliar a degradação de um Argissolo Vermelho derivado de granito, localizado na microbacia Passo do Pilão no município de Pelotas/RS, cultivado no sistema de preparo convencional e sua recuperação pelo sistema de plantio direto, comparado ao campo e à mata nativa. Em área de lavoura, foi iniciado um experimento em abril de 1995, com a semeadura de aveia preta (Avena strigosa) + ervilhaca peluda (Vicia vilosa), para implantar os sistemas de preparo convencional e plantio direto. Uma área com campo pastejado e outra com mata nativa foram amostradas para comparar a qualidade do solo com as áreas cultivadas. Foram amostradas as camadas de $0-0,05 m, 0,10-0,15 m$ e 0,20-0,25m para determinar granulometria, porosidades, densidades, retenção de água, estabilidade e distribuição do tamanho de agregados e teor de carbono orgânico. Os sistemas de cultivo aumentam a densidade e reduzem a porosidade total, a microporosidade, a retenção e a disponibilidade de água, em relação aos sistemas campo e mata nativa. O preparo convencional reduz o teor de matéria orgânica e a estabilidade dos agregados e o plantio direto, após cinco anos, aumenta pouco o teor de matéria orgânica na camada superficial, mas aumenta sensivelmente a estabilidade de agregados para valores próximos dos sistemas campo e mata nativa. A estabilidade dos agregados teve relação direta com o teor de matéria orgânica até um patamar próximo de $25 \mathrm{~g} \mathrm{~kg}^{-1}$. A densidade do solo, a macroporosidade, o parâmetro $S$, o teor de matéria orgânica e a estabilidade dos agregados são bons indicadores das mudanças que ocorrem entre os sistemas de uso do solo. No Argissolo derivado de granito, houve recuperação das propriedades relacionadas com a estabilidade da estrutura, mas nas relacionadas com densidade e porosidade do solo a recuperação não foi observada.

Palavras-chave: sistemas de manejo, estabilidade dos agregados, qualidade do solo.

\section{ABSTRACT}

Soils with high sand content in the A horizon are prone to degradation processes, depending on management system used. This study had as objective to evaluate the degradation of a Hapludalf formed from granite, located in the Passo do Pilão watershed in Pelotas county, cultivated under conventional system, and its recovery by no-tillage system, compared to two native systems, namely grass field and forest. The experiment was setup in an agricultural area, in April of 1995, with the cultivation of Avena strigosa plus Vicia vilosa, both for conventional system and no-tillage. An area under native grasses and another with native forest were sampled for comparison. Soils samples were colleted from the layers of 0 $0.05 \mathrm{~m}, 0.10-0.15 \mathrm{~m}$ and $0.20-0.25 \mathrm{~m}$, to determine the soil texture, porosity, densities, water retention, aggregate stability and distribution, and organic carbon content. The cultivation systems increase the soil bulk density and reduce total porosity, microporosity, water retention and availability, compared with native systems such as grass field and forest. The conventional system reduces organic carbon content and aggregate stability, whereas no-tillage, after five years, increases, somewhat, the organic carbon content in the surface layer, but increases aggregate stability to values near of those for native systems. The aggregate stability had direct relationship with organic carbon content up to an upper limit of $25 \mathrm{~g} \mathrm{~kg}^{-1}$. The bulk density, macroporosity, $S$ parameter, organic carbon content and aggregate stability are good indicators of the changes observed in the soil management systems. For the Hapludalf

IEmbrapa/Clima Temperado, BR 392, km 78, CP 403, 96001-970, Pelotas, RS, Brasil. E-mail: flores@cpact.embrapa.br. Autor para correspondência.

IIDepartamento de Solos, Universidade Federal de Santa Maria (UFSM), Santa Maria, RS, Brasil.

IIIDepartamento de Solos e Recursos Naturais, Universidade do Estado de Santa Catarina (UDESC), Lages, SC, Brasil.

${ }^{\text {IV }}$ Departamento de Solos, Universidade Federal de Pelotas (UFPel), Pelotas, RS, Brasil. 
formed from granite, there was recovery of the properties related to soil structure stability, but in those related to soil bulk density and porosity no recovery was observed.

Key words: management systems, aggregate stability, soil quality.

\section{INTRODUÇÃO}

No Sul do Brasil, existem extensas áreas de solos derivados de Granito, com textura superficial arenosa e relevo ondulado. A mobilização, com arações e gradagens, aumenta a porosidade, elimina plantas invasoras e incorpora matéria orgânica e nutrientes na camada mobilizada, mas degrada as propriedades químicas, físicas e biológicas do solo (COSTA et al., 2003; SILVA et al., 2006). O principal efeito é a redução da estabilidade dos agregados, o aumento da erosão hídrica e a redução da produtividade das culturas (GAERTNER et al., 2006). Especialmente nos solos arenosos, o plantio direto (PD) reduz a erosão hídrica, pela manutenção de resíduos na superfície do solo e pelo aumento no teor de carbono orgânico e de nutrientes e da capacidade de troca de cátions (BAYER \& MIELNICZUK, 1997). Além disso, aumenta a estabilidade de agregados, conforme constatado por WOHLENBERG et al. (2004), SILVA et al. (2006) e MARCOLAM \& ANGHINONI (2006), em Argissolo Vermelho, e por COSTA et al. (2003) em outras classes de solos.

Apesar dos benefícios observados no PD, geralmente, ocorre compactação na camada logo abaixo da superfície do solo, de 0,05 a 0,20m (GENRO JUNIOR et al., 2004), com redução da macroporosidade (DAM et al., 2005). Esse processo também é observado em subsuperfície no preparo convencional (PC) (COSTA et al., 2003), apesar de alguns estudos não terem encontrado diferença entre esses sistemas de manejo do solo (ALBUQUERQUE et al., 1995). Os problemas advindos com a alta compactação são: anaerobiose do solo; aumento da resistência à penetração; menor absorção de oxigênio, de água e de nutrientes; elevado contato solo/raiz; entre outros (SOANE \& VAN OUWERKERK, 1994), com sensível prejuízo às plantas quando a porosidade de aeração for inferior a $10 \%$, com difusão de oxigênio muito baixa (XU et al., 1992). Para amenizar a restrição causada pela compactação em PD, CÂMARA \& KLEIN (2005) propuseram a escarificação do solo, o que reduziu sua densidade e resistência, mas não alterou a produtividade da soja. A recuperação de propriedades físicas é observada em sistemas conservacionistas, especialmente naqueles implantados há mais tempo, mas a qualidade do solo ainda é inferior aos sistemas campo (CN) ou mata nativa (MN) (COSTA et al., 2003; WOHLENBERG et al., 2004; SILVA et al., 2006) e necessita de décadas para se aproximar da condição inicial (REINERT et al., 1990).

Este trabalho objetivou avaliar a degradação física de um Argissolo Vermelho derivado de granito cultivado no sistema de preparo convencional e sua recuperação pelo sistema de plantio direto comparado ao campo e à mata nativa.

\section{MATERIAL E MÉTODOS}

O trabalho foi conduzido na microbacia hidrográfica do Arroio Passo do Pilão, no município de Pelotas (RS), em Argissolo Vermelho Eutrófico abrúptico (EMBRAPA, 1999), com textura franco arenosa, derivado de granito. Na camada de 0 a $0,20 \mathrm{~m}$, o solo tem $160 \mathrm{~g} \mathrm{~kg}^{-1}$ de argila e $562 \mathrm{~g} \mathrm{~kg}^{-1}$ de argila, $16 \mathrm{mg} \mathrm{dm}^{-3}$ de fósforo, $144 \mathrm{mg} \mathrm{dm}^{-3}$ de potássio, 3,7 $\mathrm{cmol}$ $\mathrm{dm}^{-3}$ de cálcio, 2,2cmol $\mathrm{dm}^{-3}$ de magnésio e pH em água de 5,1. A área encontra-se na região fisiográfica da Encosta do Sudeste do Rio Grande do Sul e o experimento estava localizado nas coordenadas: Latitude $30^{\circ} 37,5^{\prime}$ " sul e Longitude $52^{\circ} 25^{\prime}$ " oeste, com altitude de 60 m. O clima da região, segundo Köppen, é Cfa2, subtropical úmido sem estiagens, com verões quentes e chuvosos com precipitação anual média entre 1.322 e 1.769mm (MORENO, 1961).

Em área anteriormente utilizada com lavouras, foi implantado um experimento, em abril de 1995, com os sistemas de plantio direto (PD) e preparo convencional (PC), em áreas adjacentes, cada uma com 0,25 ha. Para medir as condições físicas naturais desse solo, foram selecionadas mais duas áreas, uma com campo (CN) e outra com mata nativa (MN). O CN era uma área com mata que foi derrubada para ser utilizada com agricultura. Posteriormente, a área foi convertida para campo para pastoreio com gado de leite, sendo essa conversão realizada há mais de 30 anos. É composta por gramíneas e leguminosas nativas da região. A MN tem histórico de mais de 80 anos, no entanto, houve extração de “madeira de lei” antes desse período.

Na implantação, o solo foi escarificado para reduzir a compactação formada por vários anos de cultivo no sistema de PC. A acidez do solo foi corrigida com $3,6 \mathrm{Mg} \mathrm{ha}^{-1}$ de calcário e o solo foi adubado com $125 \mathrm{~kg} \mathrm{ha}^{-1}$ de $\mathrm{P}_{2} \mathrm{O}_{5}$ e 25 de $\mathrm{K}_{2} \mathrm{O}$, produtos incorporados com uma aração e duas gradagens. Foram semeadas, a lanço, plantas de cobertura consorciadas, aveia preta (Avena strigosa Schreb) + ervilhaca peluda (Vicia vilosa). No sistema de PC, as plantas de cobertura foram incorporadas com uma aração e duas gradagens e, no $\mathrm{PD}$, foram dessecadas e roladas sobre a superfície do 
solo para a semeadura do milho. Nos sistemas campo e mata nativa, o solo não recebeu adubação mineral.

Na época do pleno florescimento (fevereiro e março/2000) da cultura do milho, foram sorteados em cada sistema quatro pontos de amostragem, abertas trincheiras ( 1 x 1 x 0,6m) e coletadas amostras, em triplicatas, com estrutura preservada em anéis volumétricos e alterada em leivas, nas camadas de 0 a 0,05, 0,10 a 0,15 e 0,20 a 0,25m.

A retenção de água foi medida nas tensões de 1 e $6 \mathrm{kPa}$ em mesa de tensão e 33, 100, 500 e 1500kPa em câmara de Richards (KLUTE, 1986) e, aos pares de dados de tensão e umidade volumétrica, foi ajustada a curva de retenção de água (VAN GENUCHTEN, 1980), obtendo-se os parâmetros $\alpha$, $\eta$ e $m$. A água disponível foi calculada pela diferença entre a água retida a 10 e $1500 \mathrm{kPa}$. A porosidade total, macro e microporosidade e a densidade do solo foram determinadas pelo método do anel volumétrico (BLAKE, 1965a) e a densidade de partículas (Dp) pelo método do picnômetro (BLAKE, 1965b). Com os parâmetros $\alpha, \eta$ e $m$, foi calculado o parâmetro "S”, um índice de qualidade física do solo relacionado com a distribuição de poros por tamanho, indicativo da microestrutura do solo. A curva da umidade versus energia de retenção tem um ponto de inflexão onde a curvatura é zero e duas características particulares: sua posição $\left(\grave{e}_{\mathrm{i}}, \psi_{\mathrm{i}}\right)$ e sua inclinação $S=$ dè/ d $\psi$, obtido segundo Dexter (2004):

$$
S=-n \cdot\left(\theta_{S}-\theta_{R}\right) \cdot\left[1+\frac{1}{m}\right]^{-(1+m)}
$$

em que: $\theta_{\mathrm{s}}=$ umidade volumétrica saturada $\left(\mathrm{m}^{3} \mathrm{~m}^{-3}\right) ; \theta_{\mathrm{R}}$ = umidade volumétrica residual na tensão de $1500 \mathrm{Pka}$ $\left(\mathrm{m}^{3} \mathrm{~m}^{-3}\right) ; m$ e $n=$ parâmetros empíricos da equação de van Genuchten.

A estabilidade de agregados foi determinada em amostras de agregados entre 8,0 e 4,76mm, segundo KEMPER \& CHEPIL (1965), com peneiras de 4,76, 2,00, 1,00 e $0,25 \mathrm{~mm}$. Os agregados foram umedecidos por capilaridade por 10 minutos, agitados por 10 minutos, com 30 oscilações min $^{-1}$ e curso de oscilação vertical de $0,038 \mathrm{~m}$. O material não-gregado e retido nas peneiras foi descontado e os resultados foram expressos pelo diâmetro médio geométrico (DMG, mm). A distribuição de tamanhos de partículas foi determinada pelo método da pipeta (GEE \& BAUDER, 1986), usando-se água mais calgon e, em outra amostra, apenas a água como dispersante.

Não há um delineamento experimental clássico, pois não há casualização nas áreas CN e MN. A comparação de médias foi realizada por meio de contrastes, considerando os níveis de significância pelo teste $\mathrm{F}(\mathrm{P} \leq 0,01$ e $\mathrm{P} \leq 0,05)$ entre os sistemas: campo pastejado e mata nativa versus preparo convencional e plantio direto; campo pastejado versus mata nativa; preparo convencional versus plantio direto; e plantio direto versus mata nativa.

\section{RESULTADOS E DISCUSSÃO}

Após cinco anos de condução do experimento, as propriedades foram modificadas pelos sistemas. A densidade do solo na $\mathrm{MN}$ e $\mathrm{CN}$ foi menor do que nos cultivados, com maiores diferenças na camada de 0 a $0,05 \mathrm{~m}$. Nessa camada, a MN tem a menor densidade $\left(1,06 \mathrm{Mg} \mathrm{m}^{-3}\right)$ e o PD a maior $\left(1,49 \mathrm{Mg} \mathrm{m}^{-3}\right)$ (Tabela 1). No PC, a densidade não diferiu do PD devido á reorganização das partículas após o preparo, pois a coleta foi realizada no florescimento da cultura do milho. Geralmente, na camada superficial, é relatada maior densidade e menor macroporosidade no PD (DAM et al., 2005) e igual ou maior densidade em subsuperfície no PC (COSTA et al., 2003), enquanto outros autores (ALBUQUERQUE et al., 1995) não observaram diferenças entre os sistemas.

Os sistemas campo e mata nativa possuem maior microporosidade e porosidade total do que os cultivados, em todas as camadas. Na camada superficial, a porosidade total variou de $0,31 \mathrm{~m}^{3} \mathrm{~m}^{-3}$ no $\mathrm{PD}$ a $0,47 \mathrm{~m}^{3} \mathrm{~m}^{-3}$ na $\mathrm{MN}$ (Tabela 1 ). A MN teve maior porosidade total e macroporosidade do que o $\mathrm{CN}$ pastejado, sendo o volume de macroporos no $\mathrm{CN}$ pastejado abaixo do valor crítico de $0,10 \mathrm{~m}^{3} \mathrm{~m}^{-3}$ (XU et al., 1992) nas três camadas avaliadas, possivelmente devido ao pisoteio animal. Deve ser considerado o aumento no teor de argila na camada de 0,20 a $0,25 \mathrm{~m}$ no sistema com CN, o que contribui para aumentar a porosidade total e a microporosidade, em detrimento da macroporosidade. Nos sistemas cultivados, a macroporosidade está abaixo do valor crítico em todas as camadas, com exceção da camada superficial que foi mobilizada no PC. Apesar de a densidade nos sistemas avaliados estar abaixo do valor crítico para esta classe textural (REICHERT et al., 2003), o solo apresenta baixo volume de macroporos, com exceção da $\mathrm{MN}$, restrição que deve ser considerada no planejamento das operações de manejo do solo e das culturas para evitar degradação maior.

A retenção de água foi maior nos sistemas não-cultivados em todas as camadas e tensões (Figura 1) e no $\mathrm{CN}$ em relação à $\mathrm{MN}$ nas duas primeiras camadas, mas não diferiu entre os sistemas cultivados. A maior retenção de água está relacionada à maior microporosidade e ao teor de matéria orgânica, os quais aumentam a capilaridade e a adsorção na superfície das partículas. A disponibilidade de água, de 0 a 0,05m, 
Tabela 1 - Densidade do solo (Ds), porosidade total (PT), macro (MAC), microporosidade (MIC), massa de agregados em cada classe de tamanho e teor de matéria orgânica (MO) nas camadas, após cinco anos da implantação do experimento. Pelotas-RS, 2000.

\begin{tabular}{|c|c|c|c|c|c|c|c|c|c|c|c|}
\hline \multirow{2}{*}{ Sistemas $^{(1)}$} & \multirow{2}{*}{ Ds } & \multirow{2}{*}{ PT } & \multirow{2}{*}{ MAC } & \multirow{2}{*}{ MIC } & \multicolumn{6}{|c|}{------Massa de agregados em cada classe de tamanho------ } & \multirow{2}{*}{$\mathrm{MO}$} \\
\hline & & & & & $8,0-4,76$ & $4,76-2,0$ & $2,0-1,0$ & $1,0-0,5$ & $0,5-0,25$ & $<0,25$ & \\
\hline & $\mathrm{Mg} \mathrm{m}^{-3}$ & \multicolumn{3}{|c|}{------ $\mathrm{m}^{3} \mathrm{~m}^{-3}$} & \multicolumn{6}{|c|}{ 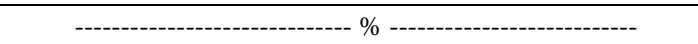 } & $\mathrm{g} \mathrm{kg}^{-1}$ \\
\hline \multicolumn{12}{|c|}{$0-0,05 \mathrm{~m}$} \\
\hline PC & 1,39 & 0,35 & 0,14 & 0,21 & 25 & 10 & 7 & 9 & 10 & 39 & 20 \\
\hline PD & 1,49 & 0,32 & 0,08 & 0,23 & 73 & 8 & 3 & 3 & 3 & 11 & 23 \\
\hline $\mathrm{CN}$ & 1,15 & 0,46 & 0,08 & 0,38 & 77 & 9 & 1,0 & 0,5 & 0,3 & 12 & 62 \\
\hline $\mathrm{MN}$ & 1,06 & 0,48 & 0,19 & 0,29 & 80 & 7 & 0,9 & 0,6 & 0,5 & 11 & 47 \\
\hline \multicolumn{12}{|c|}{ Contraste $^{(2)}$} \\
\hline MN-CN X PC-PD ${ }^{(2)}$ & $* *(3)$ & $* *$ & ns & $* *$ & $* *$ & ns & $* *$ & $* *$ & $* *$ & $* *$ & $* *$ \\
\hline MN X CN & ns & ns & $* *$ & $* *$ & ns & ns & ns & ns & ns & ns & $*$ \\
\hline PC X PD & ns & ns & $*$ & ns & $* *$ & ns & $* *$ & $* *$ & $* *$ & $* *$ & ns \\
\hline MN X PD & $* *$ & $* *$ & $*$ & $*$ & ns & ns & $* *$ & $*$ & ns & ns & $* *$ \\
\hline \multicolumn{12}{|c|}{$0,10-0,15 \mathrm{~m}$} \\
\hline PC & 1,50 & 0,33 & 0,09 & 0,23 & 51 & 14 & 5 & 6 & 5 & 19 & 17 \\
\hline PD & 1,51 & 0,31 & 0,08 & 0,23 & 45 & 12 & 7 & 9 & 7 & 20 & 14 \\
\hline $\mathrm{CN}$ & 1,51 & 0,33 & 0,05 & 0,27 & 77 & 7 & 1,0 & 0,7 & 0,6 & 14 & 23 \\
\hline $\mathrm{MN}$ & 1,33 & 0,39 & 0,14 & 0,25 & 59 & 12 & 4 & 4 & 3 & 18 & 18 \\
\hline \multicolumn{12}{|c|}{ Contraste } \\
\hline MN-CN X PC-PD & $*$ & $*$ & ns & $* *$ & $* *$ & $* *$ & $* *$ & $* *$ & $* *$ & ns & $* *$ \\
\hline MN X CN & $* *$ & $*$ & $* *$ & ns & $*$ & $*$ & $*$ & $*$ & ns & ns & $* *$ \\
\hline PC X PD & ns & ns & ns & ns & ns & ns & ns & ns & ns & ns & $*$ \\
\hline MN X PD & $* *$ & $* *$ & $*$ & ns & ns & ns & $* *$ & $* *$ & $*$ & ns & * \\
\hline \multicolumn{12}{|c|}{$0,20-0,25 \mathrm{~m}$} \\
\hline PC & 1,49 & 0,32 & 0,09 & 0,23 & 52 & 13 & 6 & 7 & 5 & 17 & 14 \\
\hline PD & 1,47 & 0,32 & 0,07 & 0,24 & 28 & 14 & 10 & 14 & 10 & 24 & 13 \\
\hline $\mathrm{CN}$ & 1,29 & 0,39 & 0,04 & 0,35 & 64 & 11 & 4 & 3 & 1,3 & 17 & 22 \\
\hline MN & 1,34 & 0,38 & 0,07 & 0,31 & 44 & 14 & 8 & 9 & 5 & 21 & 18 \\
\hline \multicolumn{12}{|c|}{ Contraste } \\
\hline MN-CN X PC-PD & $* *$ & $* *$ & ns & $* *$ & $*$ & ns & ns & $*$ & $* *$ & ns & $* *$ \\
\hline MN X CN & ns & ns & ns & ns & $*$ & ns & $*$ & $*$ & ns & ns & $* *$ \\
\hline PC X PD & ns & ns & ns & ns & $*$ & ns & $*$ & $*$ & $* *$ & ns & ns \\
\hline MN X PD & $*$ & $*$ & ns & $*$ & ns & ns & ns & ns & $* *$ & ns & $* *$ \\
\hline
\end{tabular}

(1) $\mathrm{PC}=$ Preparo convencional, $\mathrm{PD}$ = plantio direto, $\mathrm{CN}=$ campo pastejado, $\mathrm{MN}$ = mata nativa.

(2) Contrastes definidos entre os sistemas.

(3) ** significativo a 1 e * a 5 \%; ns = não-significativo.

foi maior no $\mathrm{CN}\left(0,15 \mathrm{~m}^{3} \mathrm{~m}^{-3}\right)$; a $\mathrm{MN}$ teve disponibilidade intermediária $\left(0,10 \mathrm{~m}^{3} \mathrm{~m}^{-3}\right)$; e os sistemas cultivados tiveram menor disponibilidade $(\mathrm{PD}=0,09$ e $\mathrm{PC}=0,07 \mathrm{~m}^{3} \mathrm{~m}^{-3}$ ). Entre o CN e os sistemas cultivados, a diferença foi de $50 \%$. De 0,10 a $0,15 \mathrm{~m}$ e de 0,20 a $0,25 \mathrm{~m}$ a água disponível foi menor, com média de $0,11 \mathrm{~m}^{3} \mathrm{~m}^{-3}$ para o CN, $0,085 \mathrm{~m}^{3} \mathrm{~m}^{-3}$ para a $\mathrm{MN}, 0,065 \mathrm{~m}^{3} \mathrm{~m}^{-3}$ para o PD e PC, o que indica que a compactação reduz os macroporos e os poros que retêm água disponível.

As diferenças na distribuição do tamanho dos poros e na retenção de água modificaram a inclinação da curva de retenção no ponto de inflexão (parâmetro S). Segundo DEXTER (2004), quanto maior for o módulo de $\mathrm{S}$ melhor é a qualidade do solo, sendo o valor de 0,035 o limite entre solos com pobre ou boa qualidade física. Assim, observa-se que, na camada de 0 a $0,05 \mathrm{~m}$, todos os sistemas apresentaram o módulo de $\mathrm{S}$ superior a 0,035 . Contudo, na camada de 0,10 a 0,15m, o PD e o CN, e de 0,20 a 0,25m, todos os sistemas apresentaram módulo de S menor que 0,035 (Figura 2) e, portanto, restrições físicas. O módulo de $S$ apresentou relação direta com a macroporosidade $(r=0,90 ; p=0,001)$ e indireta com a densidade do solo $(r=0,58 ; \mathrm{p}=0,05)$. Assim, amostras de sistemas com maior macroporosidade e menor densidade do solo tiveram maior módulo de $\mathrm{S}$ e melhores condições físicas. Ao estudarem os efeitos do manejo em um Calcisol Háplico do México, por meio do valor S, OSUNA-CEJA et al. 


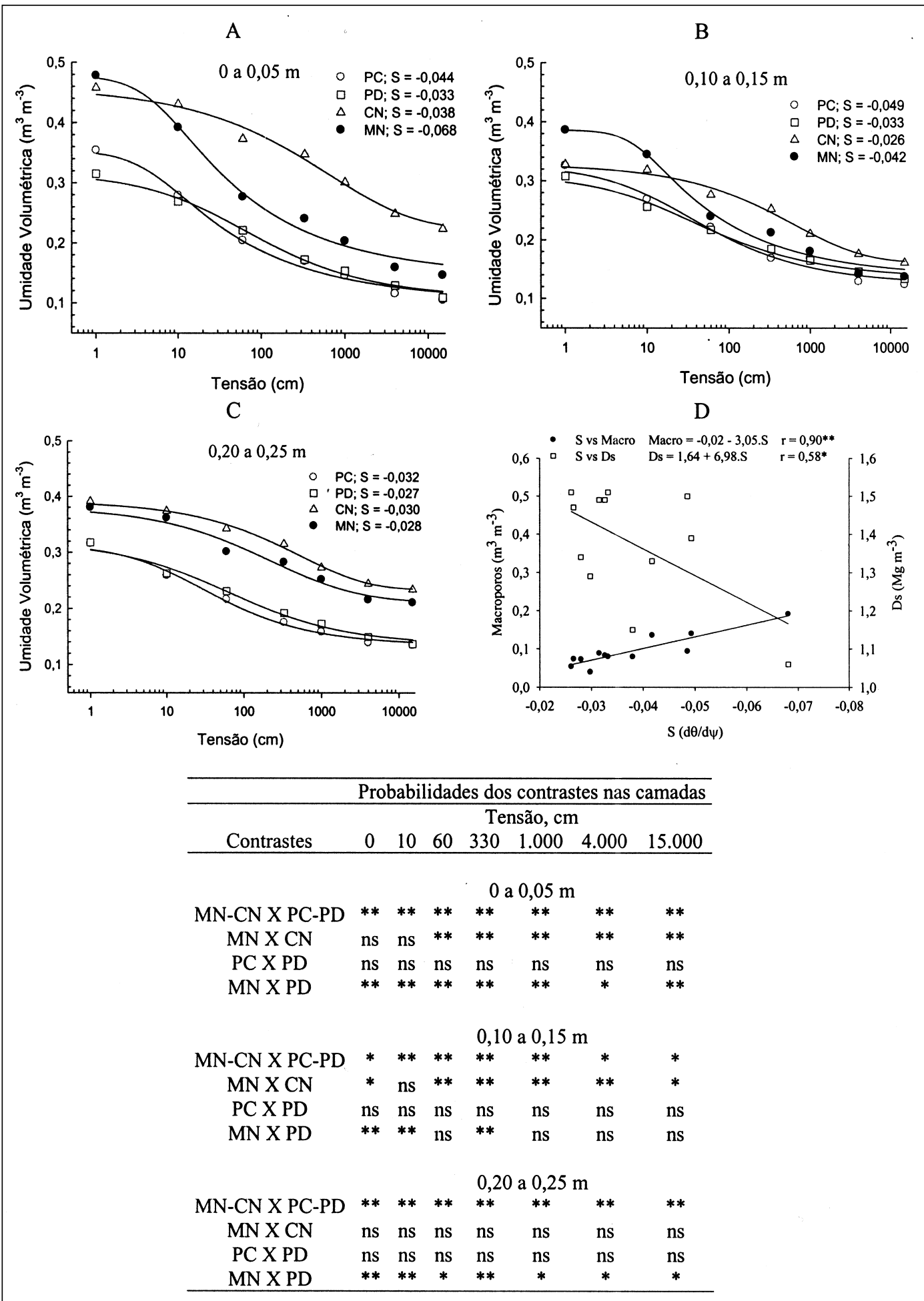

Figura 1 - Umidade volumétrica retida em diferentes tensões nas camadas de 0 a $0,05 \mathrm{~m}(\mathrm{~A}), 0,10$ a $0,15 \mathrm{~m}$ (B) e 0,20 a 0,25 m (C) e relação entre e macroporosidade e a densidade do solo (Ds) com o parâmetro $S$ (D) proposto por DEXTER (2004) nos sistemas de preparo convencional (PC), plantio direto (PD), campo pastejado (CN) e mata nativa (MN) no Argissolo Vermelho. O valor de S, na legenda, indica a inclinação da curva ajustada no ponto de inflexão. No quadro, estão definidos os contrastes com as probabilidades que indicam as diferenças significativas a $1 \%(* *)$ e a $5 \%\left(^{*}\right)$. 

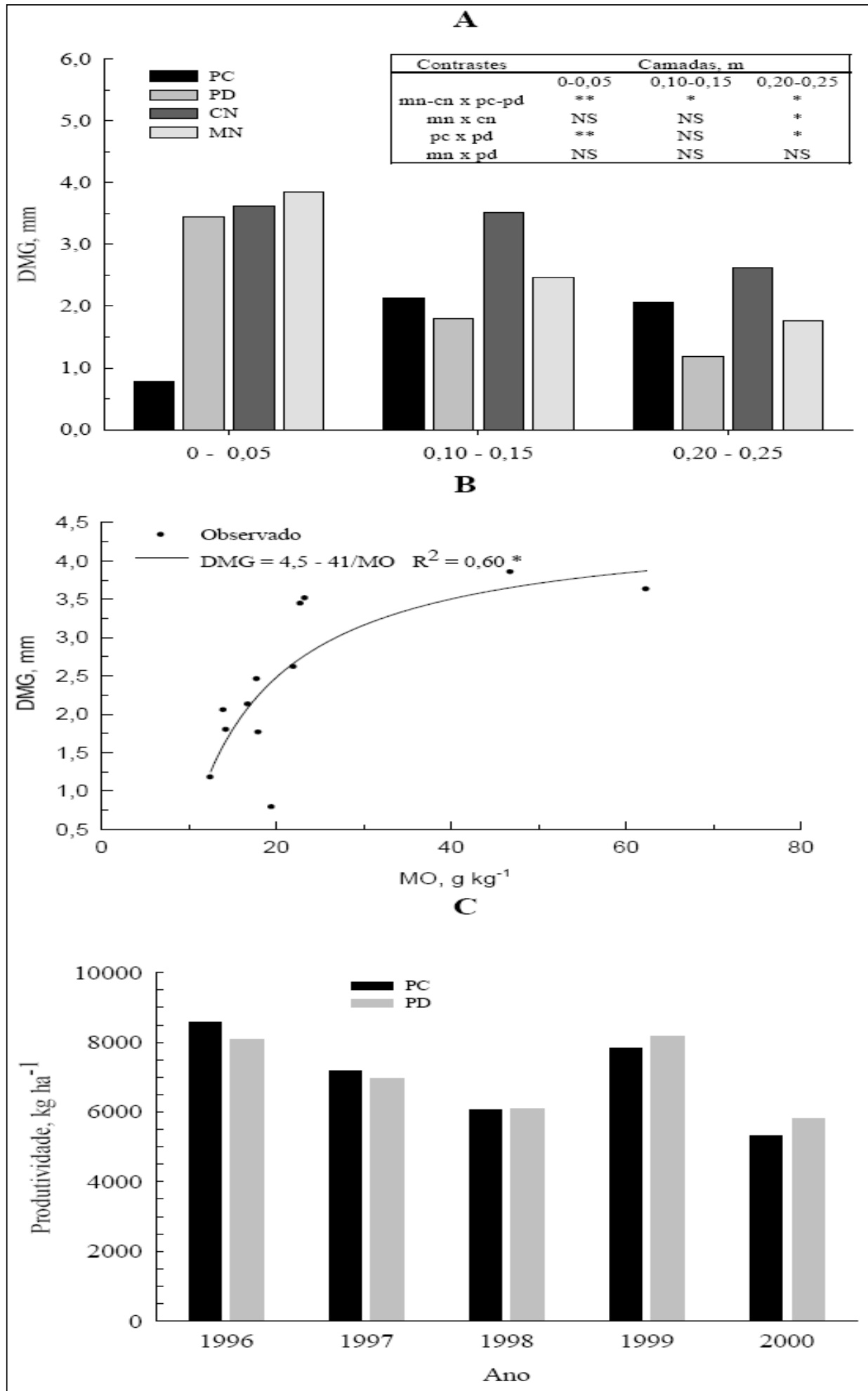

Figura 2 - Estabilidade de agregados, representada pelo diâmetro médio geométrico (DMG), nos sistemas de preparo convencional (PC), plantio direto (PD), campo pastejado (CN) e mata nativa (MN), nas três camadas avaliadas (A), sua relação com o teor de matéria orgânica (B) e produtividade da cultura do milho no período de 1996 a 2000, nos sistema de preparo convencional e plantio direto (C) do Argissolo Vermelho, Pelotas, RS. No quadro, estão definidos os contrastes com as probabilidades que indicam as diferenças significativas a $1 \%$ $(* *)$ e a $5 \%(*) . n=12$. * Significativo a $5 \%$. 
(2006), observaram melhor qualidade do preparo reduzido em relação ao PC.

A distribuição dos agregados em classes de tamanho não diferiu entre os sistemas $\mathrm{CN}$ e $\mathrm{MN}$, na camada de 0 a 0,05m, mas diferiu de 0,10 a 0,15 e 0,20 a $0,25 \mathrm{~m}$ (Tabela 1 ), tendo o $\mathrm{CN}$ pastejado maior quantidade de agregados na classe de 4,76 a $8,0 \mathrm{~mm}$, possivelmente pelo efeito agregante do sistema radicular das gramíneas, fasciculado, abundante e bem distribuído (SILVA\& MIELNICZUK, 1997). De 0a 0,05m, com mesma estabilidade, pode ter prevalecido o efeito agregante da matéria orgânica, com teor elevado para este tipo de solo e clima, nos dois sistemas ( $\mathrm{CN}=62 \mathrm{~g} \mathrm{~kg}^{-1}$ e $\left.\mathrm{MN}=47 \mathrm{~g} \mathrm{~kg}^{-1}\right)$. Mesmo sem mobilização do solo, o efeito da agitação em água, que simula o escoamento de água sobre o solo, quebrou os agregados da classe de 4,76 a $8,0 \mathrm{~mm}$, aproximadamente em 20,40 e $56 \%$ na MN e 23, 25 e $33 \%$ no CN pastejado, respectivamente nas camadas de 0 a $0,05,0,10$ a 0,15 e 0,20 a $0,25 \mathrm{~m}$ (Tabela 1). Isso indica a fragilidade do solo, que necessita ser manejado adequadamente para evitar sua degradação.

O cultivo do solo em relação aos sistemas campo e mata reduziu a quantidade de agregados maiores e aumentou a dos menores, observado, especialmente, no PC (Tabela 1). Isso se deve ao grau de mobilização do solo, maior no PC e menor no PD. Em geral, a estabilidade dos agregados nos sistemas agrícolas com mobilização do solo é menor do que na MN (COSTA et al., 2003; ALBUQUERQUE et al., 2005), e CN (CRUZ et al., 2003; SILVA et al., 2006). A mobilização do solo, durante a operação agrícola, quebra os agregados e estimula a decomposição da matéria orgânica pelos microrganismos, processo que reduz a quantidade de substâncias agregantes e, assim, a estabilidade dos agregados.

$\mathrm{O} \mathrm{PD}$, comparado ao PC, teve maior estabilidade de agregados da classe de 4,76 a $8,0 \mathrm{~mm}$, de 0 a $0,05 \mathrm{~m}$ ( 73 versus $25 \%$ ), não diferiu de 10 a $0,15 \mathrm{~m}$ ( 45 versus $51 \%$ ) e teve menor na camada de 20 a $0,25 \mathrm{~m}$ (28 versus $52 \%$ ) (Tabela 1 ). Na camada superficial, essas diferenças modificaram a estabilidade dos agregados, tendo os sistemas MN e CN, com diâmetro médio geométrico (DMG) de 3,9 e 3,6mm respectivamente, maior estabilidade do que os cultivados (Figura 2) e a menor estabilidade no PC (DMG de 0,8mm), com redução de $80 \%$ em relação à $\mathrm{MN}$. No PD, houve recuperação da estabilidade dos agregados (DMG de 3,4mm) em $330 \%$, comparado ao PC, e alcançou 90\% da estabilidade da MN, modificação também constatada por COSTA et al. (2003). Nas camadas inferiores, as diferenças foram menores entre sistemas, mas, na camada de 0,20 a 0,25m, o PC teve maior estabilidade do que o PD. No PC, a quantidade de MO foi estável com a profundidade devido à incorporação pela lavração, enquanto no PD houve maior teor na camada superficial e menor nas camadas mais profundas, como constatado também por BAYER \& MIELNICZUK (1997) e SILVA et al. (2006). Menor estabilidade dos agregados pelo uso do PC em relação ao PD tem sido freqüentemente constatada (CARPENEDO \& MIELNICZUK, 1990; COSTA et al., 2003; SILVA et al., 2006), especialmente nos solos arenosos (SILVA et al., 2006). No PC, a manutenção dos resíduos na superfície e o uso de arado de aiveca, comparado ao de disco, reduzem os efeitos negativos na estabilidade dos agregados (ALVARENGA et al., 1986). Em Argissolo Vermelho, a estabilidade dos agregados não foi modificada pelo manejo, provavelmente pelo pequeno tempo de uso, três anos, do sistema de PD (CRUZ et al., 2003); portanto, o histórico e o tempo de uso são fatores que interferem na qualidade do solo.

Em observação visual, os agregados nos sistemas naturais caracterizaram-se pela forma mais arredondada, enquanto que nos sistemas agrícolas, principalmente no sistema convencional, foram mais arestados. Essa morfologia, associada à baixa estabilidade na camada de 0 a $0,05 \mathrm{~m}$ de profundidade, no sistema de PC, permite inferir que sua formação pode ter sido decorrente do fracionamento de agregados grandes e de forças compressivas provocadas pelo tráfego de máquinas e implementos agrícolas. Essa constatação foi caracterizada em exame micromorfológico em solos do Rio Grande do Sul por CARPENEDO \& MIELNICZUK (1990).

O teor de matéria orgânica foi elevado nos sistemas CN e MN, enquanto a mobilização do solo no PC reduziu em $60 \%$ esse teor em relação à MN (Tabela 1). Após cinco anos de implantação do experimento, o teor médio de matéria orgânica foi de $17 \mathrm{~g} \mathrm{~kg}^{-1}$, tendo o PD acumulado mais matéria orgânica de 0 a $0,05 \mathrm{~m}$, enquanto no PC não teve diferença entre camadas. Em solos de textura arenosa, de menor área superficial específica, e nas regiões de clima mais quente, a taxa de decomposição da matéria orgânica é maior do que nos argilosos e no clima mais frio (SIX et al., 2002), o que dificulta o acúmulo de matéria orgânica no solo, principalmente em sistemas agrícolas. Em Argissolo Vermelho franco arenoso e região de clima subtropical, BAYER et al. (2000) observaram que o PD teve a metade da taxa de perda de matéria orgânica em comparação com o PC, e o acúmulo de matéria orgânica, após 15 anos, só foi possível com a utilização de sistemas de culturas com grande adição de resíduos.

O acúmulo de matéria orgânica no PD, na camada de 0 a $0,05 \mathrm{~m}$, aumentou a estabilidade dos 
agregados, indicando que, nesse solo, a matéria orgânica pode ser considerada o principal agente de agregação, já que o processo de decomposição pelos microrganismos produz agentes de ligação (MUNNER \& OADES, 1989). Relação entre matéria orgânica e estabilidade de agregados tem sido relatada por vários autores (LE BISSONNAIS \& ARROUAYS, 1997; SIX et al., 2002; SILVA et al., 2006). Nesse solo, com baixo teor de argila e mineralogia caulinítica, a matéria orgânica é o principal agente de agregação, tendo o sistema radicular efeito importante (SILVA \& MIELNICZUK, 1997; TISDAL \& OADES, 1982). Esses autores citam que os agregados maiores que $2,0 \mathrm{~mm}$ são mantidos unidos principalmente pelo efeito agregante das hifas e raízes, mas é transitório e muito sensível ao preparo do solo. Nesse Argissolo, existe um patamar, próximo de $25 \mathrm{~g} \mathrm{~kg}^{-1}$, a partir do qual o aumento da matéria orgânica, aparentemente, não se traduz em aumento da estabilidade dos agregados (Figura 2). Em solos siltosos do sudoeste da França, com histórico de cultivo entre oito e 100 anos, além do efeito na estabilidade, LE BISSONNAIS \& ARROUAYS (1997) constataram que o aumento no teor de matéria orgânica até 15 a $20 \mathrm{~g} \mathrm{~kg}^{-1}$ teve relação direta com a infiltração de água no solo. Assim, pequenos aumentos no teor de matéria orgânica aumentam a estabilidade dos agregados e favorecem os processos relacionados.

A produtividade do milho de 1996 a 2000 não diferiu entre o PC e PD, com oscilações entre os anos em função da variabilidade climática (Figura 2). O PC teve maior produtividade nos dois primeiros anos, enquanto o PD produziu mais nos três últimos cultivos. Assim, o PD, após sua consolidação como sistema, poderá produzir mais que o PC, conforme observado por COSTA et al. (2003) em Latossolo Bruno, enquanto MARCOLAN \& ANGHINONI (2006) em Argissolo Vermelho e CÂMARA \& KLEIN (2005) em Latossolo Vermelho não observaram diferenças entre os sistemas PC e PD.

\section{CONCLUSÕES}

Os sistemas de preparo convencional e plantio direto aumentam a densidade e reduzem a porosidade total, a microporosidade, a retenção e a disponibilidade de água em relação ao campo e à mata nativa em Argissolo Vermelho. O preparo convencional reduz o teor de matéria orgânica e a estabilidade dos agregados, enquanto o plantio direto, após cinco anos, aumenta o teor de matéria orgânica na camada superficial e eleva a estabilidade de agregados para valores próximos dos sistemas campo e mata nativa. A estabilidade dos agregados tem relação direta com o teor de matéria orgânica até um patamar próximo de $25 \mathrm{~g} \mathrm{~kg}^{-1}$, a partir do qual o aumento da matéria orgânica não se traduz em aumento da estabilidade dos agregados. A densidade do solo, a macroporosidade, o parâmetro S, o teor de matéria orgânica e a estabilidade dos agregados são bons indicadores das mudanças que ocorrem no solo. No Argissolo, o plantio direto recupera a estabilidade da estrutura, observada pelo aumento do diâmetro médio geométrico, mas não os atributos relacionados à densidade e à porosidade do solo.

\section{REFERÊNCIAS}

ALBUQUERQUE, J.A. et al. Rotação de culturas e sistemas de manejo do solo. I- Efeito sobre a forma da estrutura do solo ao final de sete anos. Revista Brasileira de Ciência do Solo, v.19, n.1, p.115-119, 1995

ALBUQUERQUE, J.A. et al. Relação de atributos do solo com a agregação de um Latossolo Vermelho sob sistemas de preparo e plantas de verão para cobertura do solo. Revista Brasileira de Ciência do Solo, v.29, n.3, p.415-424, 2005.

ALVARENGA, R.C. et al. Estabilidade de agregados de um Latossolo Roxo sob diferentes métodos de preparo do solo e de manejo da palhada de milho. Revista Brasileira de Ciência do Solo, v.10, p.273-277, 1986.

BAYER, C.; MIELNICZUK, J. Características químicas do solo afetadas por métodos de preparo e sistemas de cultura. Revista Brasileira de Ciência do Solo, v.21, n.1, p.105112, 1997.

BAYER, C. Efeito de sistemas de preparo e de cultura na dinâmica da matéria orgânica e na mitigação das emissões de $\mathrm{CO}_{2}$. Revista Brasileira de Ciência do Solo, v.24, n.3, p.599-607, 2000.

BLAKE, G.R. Bulk density. In: BLACK, C.A. (Ed.). Methods of soil analysis; physical and mineralogical properties, including statistics of measurement and sampling. Madison: American Society of Agronomy. 1965a. p.374-390.

BLAKE, G.R. Particle density. In: BLACK, C.A. (Ed.). Methods of soil analysis: physical and mineralogical properties, including statistics of measurement and sampling. Madison: American Society of Agronomy, 1965b. p.371-373.

CAMARA, R.K.; KLEIN, V.A. Propriedades físico-hídricas do solo sob plantio direto escarificado e rendimento da soja. Ciência Rural, v.35, n.4, p.813-819, 2005.

CARPENEDO, V.; MIELNICZUK, J. Estado de agregação e agregados de Latossolos Roxos submetidos a diferentes sistemas de manejo. Revista Brasileira de Ciência do Solo, v.14, n.1, p.99-105, 1990.

COSTA, F.S. et al. Propriedades físicas de um Latossolo Bruno afetados pelos sistemas plantio direto e preparo convencional. Revista Brasileira de Ciência do Solo, v.27, n.3, p.527535, 2003 
CRUZ, A.C.R. et al. Atributos físicos e carbono orgânico de um Argissolo Vermelho sob sistemas de manejo. Revista Brasileira de Ciência do Solo, v.27, n.6, p.1105-1112, 2003.

DAM, R.F. et al. Soil bulk density and crop yield under eleven consecutive years of corn with different tillage and residue practices in a sandy loam soil in central Canada. Soil \& Tillage Research, v.84, n.1, p.41-53, 2005.

DEXTER A.R. Soil physical quality: Part I. Theory, effects of soil texture, density, and organic matter, and effects on root growth. Geoderma, v.120, n.3-4, p.201-214, 2004.

EMPRESA BRASILEIRA DE PESQUISA AGROPECUÁRIA. Centro Nacional de Pesquisa de Solos. Sistema Brasileiro de Classificação de Solos. Brasília: Embrapa, Serviço de Produção de Informação, 1999. 412p.

GAERTNER, C. et al. Produtividade do trigo e da soja em Latossolo Vermelho distrófico com diferentes níveis de erosão hídrica. Scientia Agraria, v.7, n.1-2, p.27-34, 2006.

GEE, G.W.; BAUDER, J.W. Particle-size analysis. In: KLUTE, A. (Ed.). Methods of soil analysis. Physical and mineralogical methods. 2.ed. Madison: American Society of Agronomy, 1986. p.383-411.

GENRO JUNIOR, S.A. et al. Variação temporal da resistência à penetração do solo de um Latossolo argiloso sob semeadura direta com rotação de culturas. Revista Brasileira de Ciência do Solo, v.28, n.3, p.477-484, 2004.

KEMPER, W.D.; CHEPIL, W.S. Size distribution of aggregates. In: BLACK C.A. et al. (Eds.). Methods of soil analysis: physical and mineralogical methods. Madison: Am Soc Agr, 1965. p.499-510.

KLUTE, A. Water retention: laboratory methods. In: KLUTE, A. (Ed.). Methods of soil analysis: physical and mineralogical methods. 2.ed. Madison: American Society of Agronomy, 1986. p.635-662.

LE BISSONNAIS, L.; ARROUAYS, D. Aggregate stability and assessment of soil crustability and erodibility: II. Application to humic loamy soils with various organic carbon contents. European Journal of Soil Science, v.48, n.1, p.39-48, 1997.

OSUNA-CEJA, E.S. et al. Efecto de la estructura del suelo sobre el desarrollo radical del maíz con dos sistemas de labranza. Agrociência, v.40, n.1, p.27-38, 2006.

MARCOLAN, A.L.; ANGHINONI, I. Atributos físicos de um Argissolo e rendimento de culturas em função do revolvimento do solo em plantio direto. Revista Brasileira de Ciência do Solo, v.30, n.1, p.163-170, 2006.

MORENO, J.A. Clima do Rio Grande do Sul. Porto Alegre: Secretaria da Agricultura, Diretoria de Terras e Colonização, Seção de Geografia, 1961. 46p.

MUNNER, M.; OADES, J.M. The role of Ca-organic interactions in soil aggregate stability. III. Mechanics and models. Australian Journal of Soil Research, v.27, n.2, p.411423, 1989.

REICHERT, J.M. et al. Qualidade dos solos e sustentabilidade de sistemas agrícolas. Ciência \& Ambiente, v.27, n.2, p.29$48,2003$.

REINERT, D.J. et al. Temporal variation in structural stability induced by tillage. In: EASTERN CANADA SOIL STRUCUTRE WORKSHOP, 1990, Guelph, Ontario. Proceedings... Windsor, Ontario: Canadian Society of Soil Science, 1990. V.1. 236p. p.63-72.

SILVA, I.F.; MIELNICZUK, J. Ação do sistema radicular de plantas na formação e estabilização de agregados do solo. Revista Brasileira de Ciência do Solo, v.21, n.1, p.113117, 1997

SILVA, M.A.S. et al. Propriedades físicas e teor de carbono orgânico de um Argissolo Vermelho sob sistemas de uso e manejo. Revista Brasileira de Ciência do Solo, v.30, n.2, p.327337, 2006.

SIX, J. et al. Soil organic matter, biota and aggregation in temperate and tropical soils - Effects of no-tillage. Agronomie, v.22, p. 55-775, 2002.

SOANE, B.D.; OUWERKERK, C. van. Soil compaction problems in world agriculture. In: SOANE, B.D.; OUWERKERK, C. van. (Eds). Soil compaction in crop production. Netherlands: Elsevier, 1994. p.1-21.

TISDALL, J.M.; OADES, J.M. Organic matter and waterstable aggregates in soils. Journal of Soil Science, v.33, n.2, p.141-163, 1982.

VAN GENUCHTEN, M.T. A closed-form equation for predicting the hydraulic conductivity of unsaturated soils. Soil Science Society American Journal, v.44, n.5, p.892-898, 1980 .

WOHLENBERG, E.V. et al. Dinâmica da agregação de um solo franco-arenoso em cinco sistemas de culturas em rotação e em sucessão. Revista Brasileira de Ciência do Solo, v.28, n.5, p.891-900, 2004.

$\mathrm{XU}, \mathrm{X}$. et al. Compaction effect on the gas diffusion coefficient in soils. Soil Science Society American Journal, v.56, n.6, p.1743-1750, 1992. 